\title{
Research on Power of Resonant Wireless Power Transfer System at Different Distances
}

\author{
Jianliang $\mathrm{Li}^{1}$, a,* , TingChuan $\mathrm{Xie}^{1}$, Jing Kang${ }^{1}$, Yalan $\mathrm{Hao}^{2}$, Qiuyue Zhang ${ }^{1}$, \\ Yuefei $\mathrm{Ma}^{1}$
}

${ }^{1}$ School of Construction Machinery College, Chang'an University, Xi'an 710064, China.

${ }^{2}$ School of Highway, Chang'an University, Xi'an 710064, China.

$$
\text { *,a414777192@qq.com }
$$

\begin{abstract}
Resonant Wireless Power Transfer (RWPT) is free from wired transmission. It has also realized long-distance power transmission, which has become one of the hotspots at home and abroad. Based on the existing research foundation, this paper focuses on the analysis of the inverter module, rectifier module and resonant coupling module from the components of RWPT. Further, PSpice simulation is used to analyze the IR2110 driving circuit and the transmission process of the whole system. Finally, a RWPT system is set up to explore the feasibility and effectiveness of theoretical analysis and practical experiments.
\end{abstract}

Key words: RWPT; PSpice; IR2110.

\section{Rwpt System Introduction}

The discovery of RWPT can be traced back to the end of the nineteenth Century, Nicola Tesla did a lot of research on it . Then, MIT's research team really pushed RWPT into the hot spot of scientific research in 2007[1]. The components of the RWPT system are shown in FIG 1. Power frequency AC power from the power grid is changed into direct current power by rectifier circuit (AC-DC), direct current power by chopper circuit (DC-DC) and high frequency AC power by inverter circuit (DCAC). Next, high frequency electricity passes through the resonant circuit to transfer energy from the original side to the secondary side, and the secondary side is then output to the load through the rectification circuit (DC-DC) and the filter circuit [2].

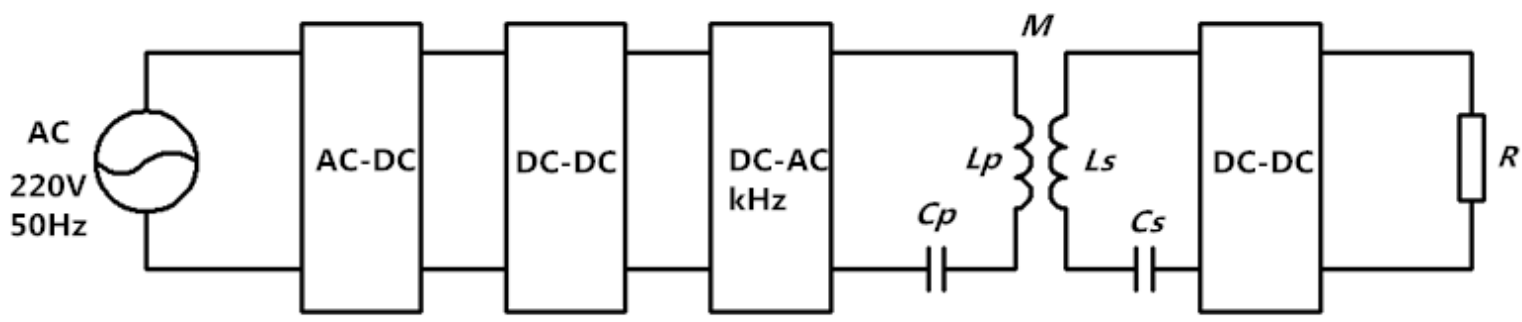

Fig. 1 Electrical Schematic for WP

\section{System Analysis}

\subsection{Module Analysis}

In this study, the DC switching power supply is used as the energy transmitting end. Therefore, the original side inverter module, the secondary side rectification filtering module and the resonance module are mainly studied. According to the existing research foundation, the inverter module uses a full-bridge inverter circuit, the rectifier module uses a full-wave uncontrollable rectifier circuit, and the resonance module uses a string-string type resonant circuit [3]. 


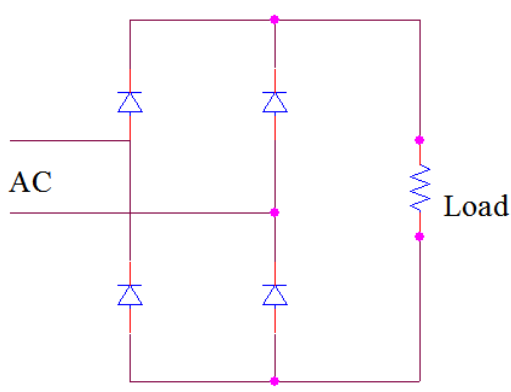

Fig. 2 Full Bridge Inverter Circuit

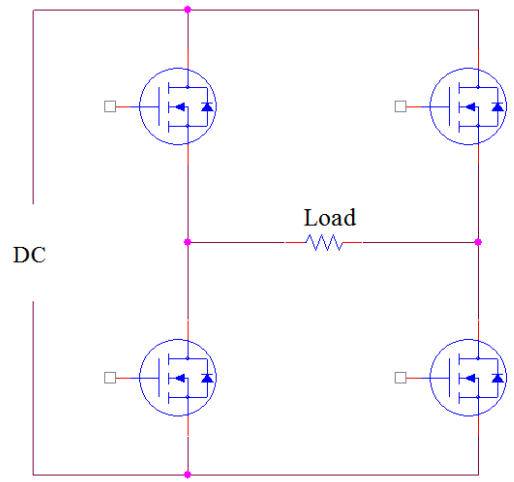

Fig. 3 Full Wave Rectifying Circuit

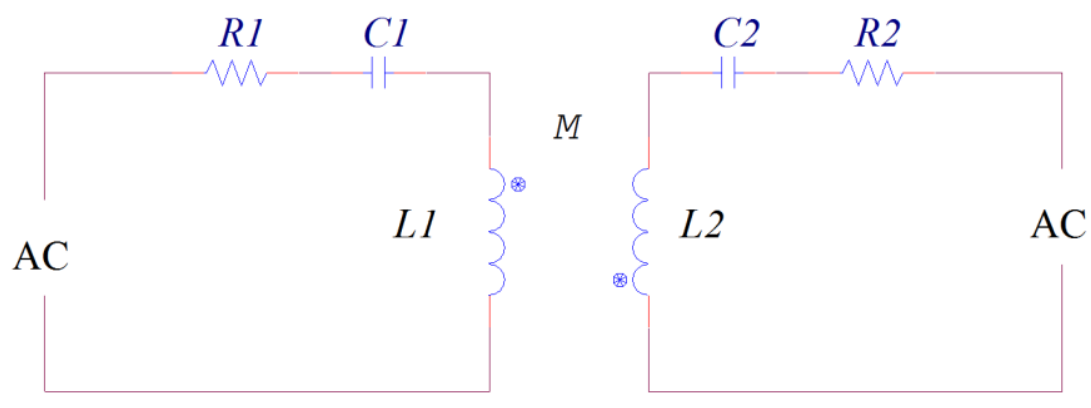

Fig.4 Serial Resonant Circuit

As shown in FIG. 2, the full-bridge inverter circuit is mainly composed of four switching tubes, and the diagonal line is one group, there are two groups in total. Under the control of the driving signal, the two sets of switching tubes are alternately turned on, thereby achieving DC-changing communication.

As shown in FIG 3, the rectifying circuit is mainly composed of four diodes, and the diagonal line is one group, which alternately passes through alternating current, rectifies into direct current, and then supplies power to the load end through a filter voltage stabilizing circuit.

As shown in FIG. 4, the resonant module is a serial resonant circuit, namely LC oscillation circuit. Among them, R1 and R2 are circuit equivalent internal resistance, $\mathrm{C} 1$ and $\mathrm{C} 2$ are resonant capacitors, $\mathrm{L} 1$ and $\mathrm{L} 2$ are resonant inductors, $\mathrm{M}$ is mutual inductance, and $\mathrm{k}$ is a coupling coefficient. The mutual relationship between mutual inductance and coupling coefficient is $M=k \sqrt{L_{1} L_{2}}$. In the power-on state, $\mathrm{L}$ and $\mathrm{C}$ produce oscillations. When the frequency of the original and secondary oscillations is the same, the resonance is produced and the resonant frequency is $f=1 / 2 \pi \sqrt{L C}$. At this time, a larger power of energy transmission can be realized.

The circuit equations of the circuit can be obtained by Kirchhoff's law and mutual inductance theory [4]:

$$
\left\{\begin{array}{l}
U=Z_{1} I_{1}-j \omega_{1} M I_{2} \\
0=Z_{2} I_{2}-j \omega_{2} M I_{1}
\end{array}\right.
$$

$\mathrm{U}$ is the power supply voltage. $I_{1}$ and $I_{2}$ are the currents of the original and secondary sides. $\omega_{1}$ and $\omega_{2}$ are the original and secondary resonant angular frequencies. Among them,

$$
\left\{\begin{array}{l}
Z_{1}=R_{1}+\frac{1}{j \omega_{1} C_{1}}+j \omega_{1} L_{1} \\
Z_{2}=R_{2}+R+\frac{1}{j \omega_{2} C_{2}}+j \omega_{2} L_{2}
\end{array}\right.
$$


In the resonant state, the resonant frequency of the original and secondary sides is the same. It behaves as $\omega=\omega_{1}=\omega_{2}$. Introduced by the resonant conditions mentioned above (the resonant conditions is $\omega=\frac{1}{\sqrt{L C}}$ ), we deduce $L_{1} C_{1}=L_{2} C_{2}$, and we know that $\omega L=\frac{1}{\omega C}$. Then we can calculate the expression of two unknowns $I_{1}, I_{2}$ in equation (1):

$$
\begin{gathered}
I_{1}=\frac{U\left(R_{2}+R\right)}{R_{1}\left(R_{2}+R\right)+(\omega M)^{2}} \\
I_{2}=\frac{j \omega M U}{R_{1}\left(R_{2}+R\right)+(\omega M)^{2}}
\end{gathered}
$$

The power calculation formula for the load is

$$
P=I^{2} R
$$

Substituting equation (4 into equation (5), the power of the load is

$$
P=\frac{(\omega M)^{2} U^{2} R}{\left[R_{1}\left(R_{2}+R\right)+(\omega M)^{2}\right]^{2}}
$$

The transmission efficiency of the system is the ratio of load power to power output power, i. e,

$$
\eta=\frac{P}{U I_{1}}=\frac{(\omega M)^{2} R}{\left[R_{1}\left(R_{2}+R\right)+(\omega M)^{2}\right]\left(R_{2}+R\right)}
$$

In order to better analyze the influence of coil coupling coefficient on system power and efficiency, the mathematical relationship was explored with software Mathcad. We assume that the power supply voltage is $12 \mathrm{~V}$, the inductance is $135 \mathrm{uh}$, the capacitance is $0.47 \mathrm{nf}$, the equivalent resistance is $0.5 \Omega$, and the calculated resonant frequency is $20 \mathrm{kHz}$. The coupling coefficient $\mathrm{K}$ is set to $0.1 \sim 1.0$ with a step length of 0.01. As shown in FIG. 5, the relationship between the total impedance and the loop current of the series resonant circuit shows that the total impedance at the resonance point is 0.473 , and the current value is at most 25.385, which is most beneficial for energy transmission. In addition, it can be seen that the frequency range which can be adjusted is small, and once it deviates from the resonance point, the impedance sharply increases, and the current suddenly drops [5]. FIG. 6 shows the relationship between the coupling coefficient $\mathrm{k}$ and efficiency and power, which increases the efficiency by 50 times for analysis. It can be seen that with increasing of the coupling coefficient, the efficiency increase firstly and then stabilizes, and the power increases firstly and then decreases. This provides a reference standard for the next step of selection.

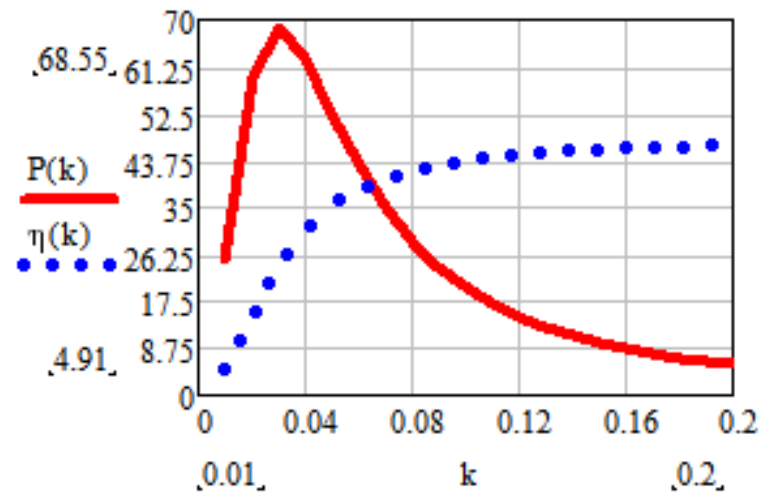

Fig. 5Relationship between Total Impedance and Current 


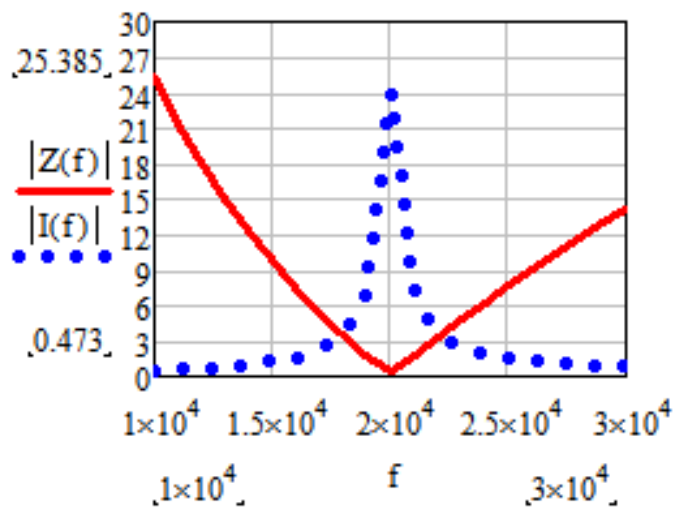

Fig. 6The Relationship between Coupling Coefficient k and Efficiency and Power

\subsection{Simulation Analysis}

This part is mainly based on the above analysis, using PSpice software to simulate inverter, rectifier and the whole system.

The $12 \mathrm{~V}$ DC power source is selected as the energy source. According to the related calculation, the inverter circuit switch tube is irf840, the continuous current diode is 1N4376, the drive chip is IR2110, each IR2110 can send 1 high level, 1 low level, and then controls the switch of the MOSFET switch.

According to the analysis of FIG. 5 and FIG. 6, the system has the best effect at the frequency of $20 \mathrm{kHz}$, and the coupling coefficient is best between 0.02 0.08.

In the IR2110 drive circuit of FIG. 7, the switching frequency is set to $20 \mathrm{kHz}$, which is consistent with the resonant frequency of the coupled part in FIG. 8, and the coupling coefficient is fixed to 0.06, in which the PWM wave uses two pulse power sources to replace the function of the TMS320F28335.

FIG. 9 is the four waveform of the two IR2110, and setting a certain dead time to prevent the short circuit of the inverting circuit from going up and down with the bridge arm.

FIG. 10 and FIG. 11 are the pickup voltage of the secondary coil and the voltage diagram of the load side after being rectified.

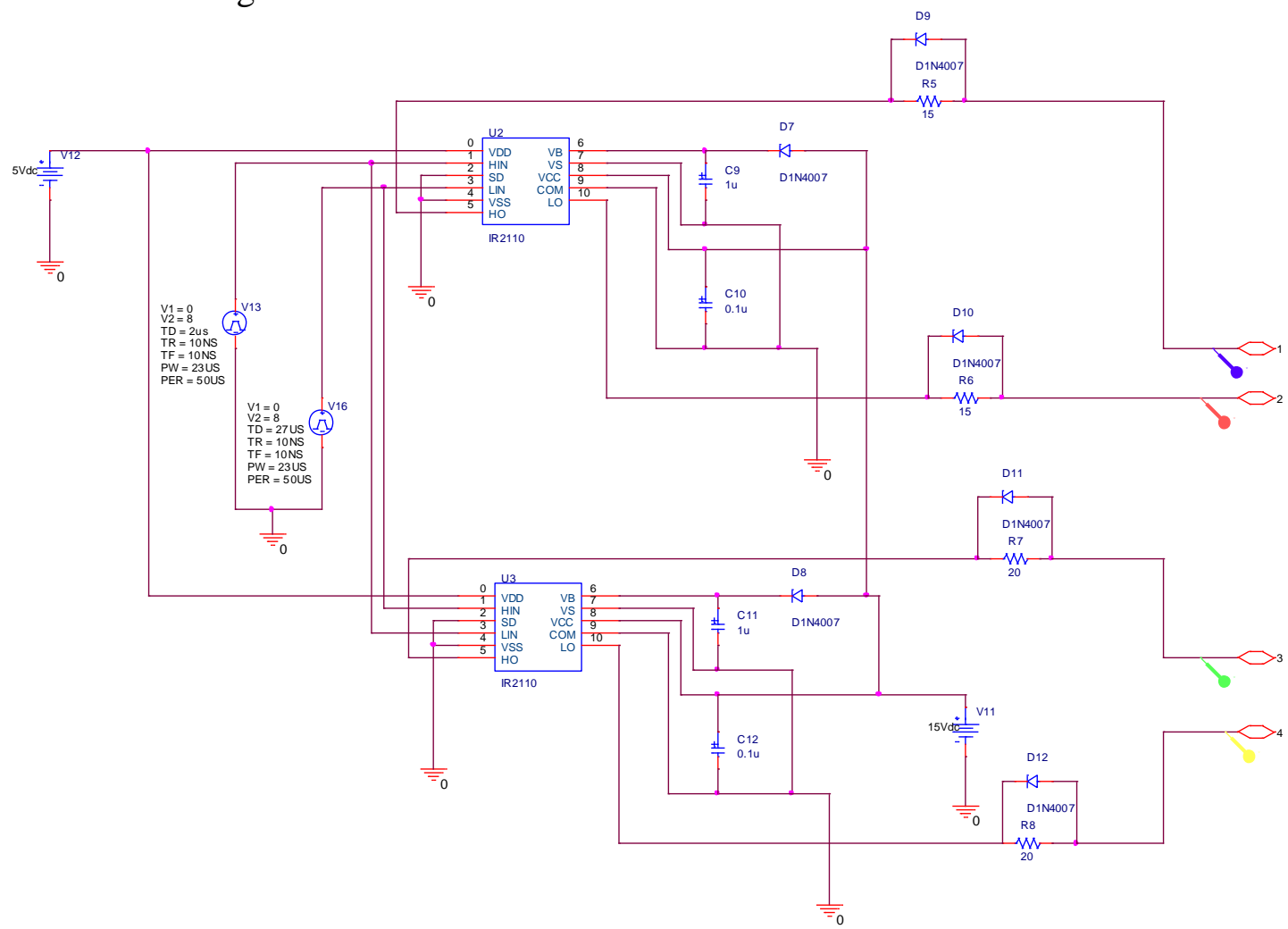

Fig. 7 IR2110 Drive Circuit 


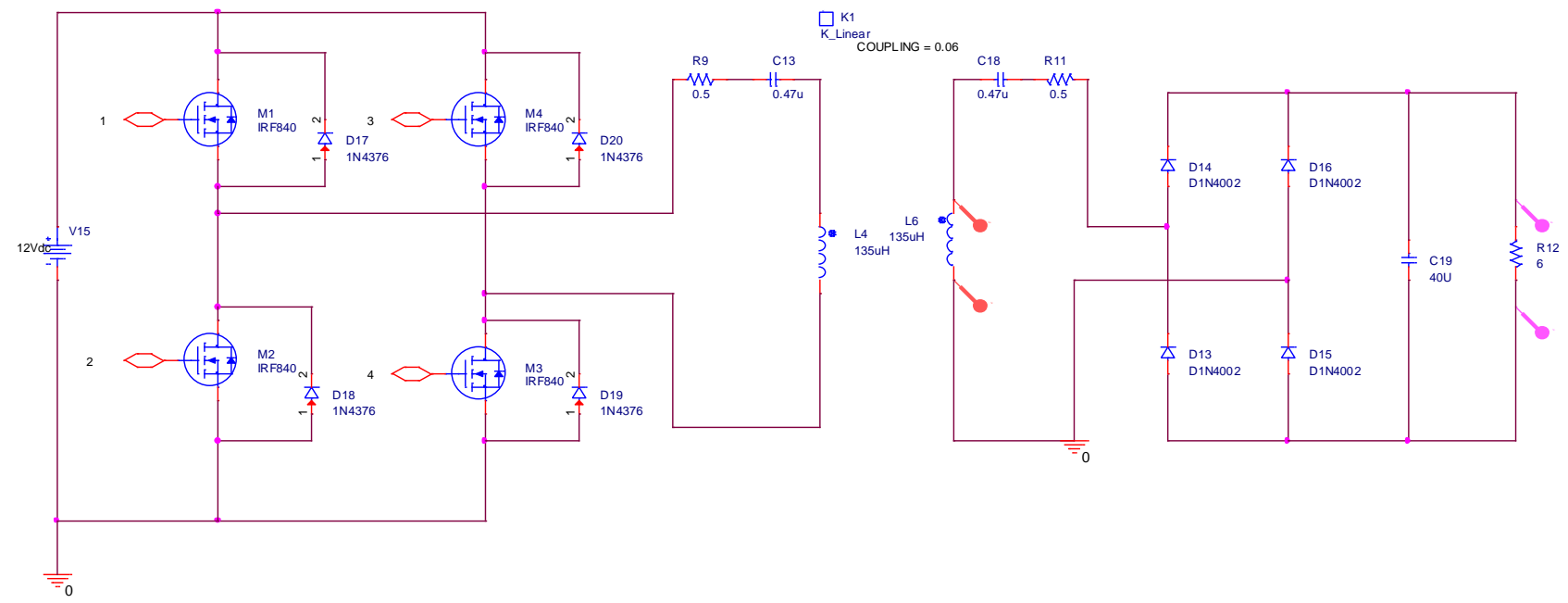

Fig. 8 System Simulation Diagram

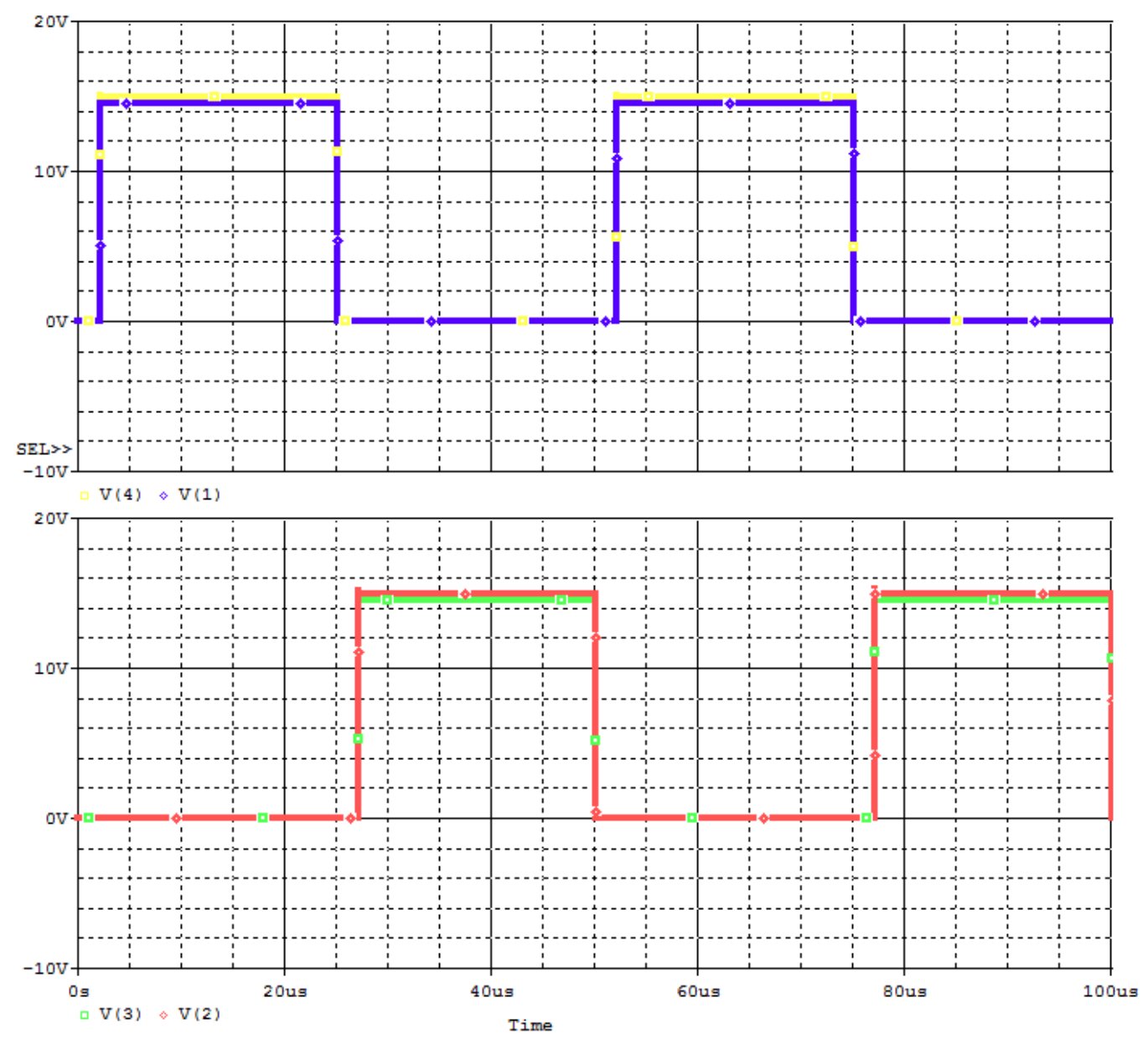

Fig. 9 IR2110 Waveform Diagram 


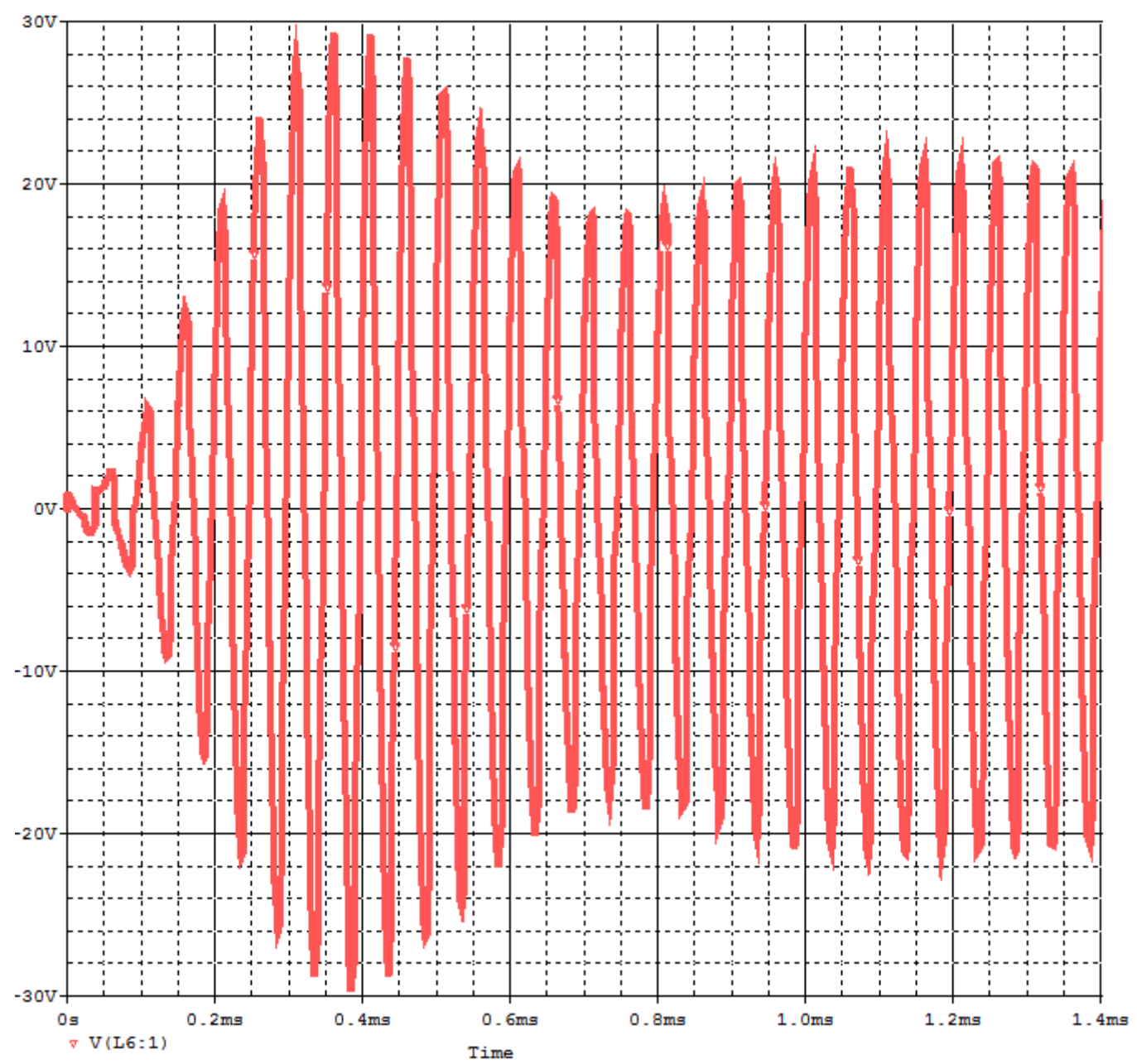

Fig. 10 Secondary Coil Voltage

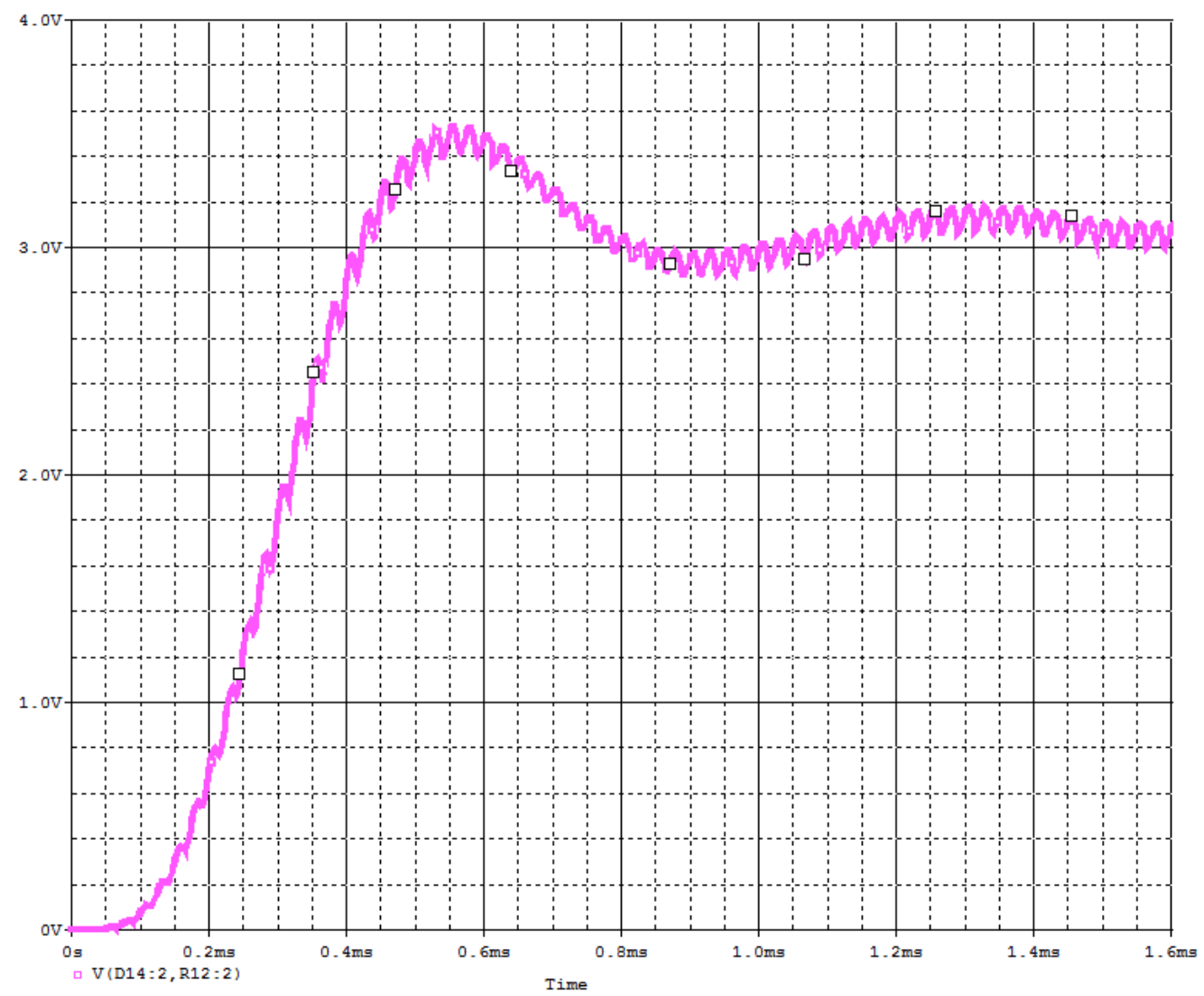

Fig. 11 Load Voltage 


\section{Experimental}

A RWPT system is designed to verify the validity and feasibility of theoretical analysis and simulation analysis. The system parameters are shown in Table 1, and the components selection is shown in Table 2.

Table 1. System Parameters

\begin{tabular}{cc}
\hline Parameter & Value \\
\hline Voltage/V & 12 \\
$L_{1} / \mu \mathrm{H}$ & 135 \\
$L_{2} / \mu \mathrm{H}$ & 135 \\
$C_{1} / \mu \mathrm{F}$ & 0.47 \\
$C_{2} / \mu \mathrm{F}$ & 0.47 \\
$\mathrm{Load} / \Omega$ & 150 \\
\hline
\end{tabular}

Table 2. Component Selection

\begin{tabular}{cc}
\hline Component & Model \\
\hline MOSFET & irf840 \\
Fly-wheel diode & $1 \mathrm{~N} 4148$ \\
Commutation diode & D1N5820 \\
Filter capacitor & $4.7 \mathrm{uF}$ \\
Inverter control chip & IR2110 \\
DSP & TMS320F28335 \\
\hline
\end{tabular}

The degree of coupling between the two sides of the coil is smaller and smaller with the increasing of the distance of the coil, and the experiment is based on the different spacing of the coil. The distance between the coils is $2 \mathrm{~cm}, 3 \mathrm{~cm}, 4.5 \mathrm{~cm}, 6 \mathrm{~cm}, 9 \mathrm{~cm}, 12 \mathrm{~cm}, 15 \mathrm{~cm}, 18 \mathrm{~cm}, 9 \mathrm{~cm}$. The load range is $0 \sim 100 \Omega$. The $6 \mathrm{~cm}$ distance experiment data is shown in Table 3.

Table 3. Experimental data from $6 \mathrm{~cm}$

\begin{tabular}{cccc}
\hline Load/ $\Omega$ & Voltage/V & Current/A & Power/W \\
\hline 1.5 & 2.62 & 1.27 & 3.3274 \\
4.4 & 3.86 & 1.92 & 7.4112 \\
8.7 & 6.15 & 1.95 & 11.9925 \\
11.8 & 6.87 & 2.05 & 14.0835 \\
14.5 & 7.26 & 2.05 & 14.883 \\
18.4 & 8.15 & 1.92 & 15.648 \\
24.9 & 8.17 & 2.06 & 16.8302 \\
32.4 & 8.58 & 2.01 & 17.2458 \\
55.3 & 8.91 & 2.01 & 17.9091 \\
70 & 9.35 & 2.04 & 19.074 \\
105.5 & 9.42 & 2.06 & 19.4052 \\
\hline
\end{tabular}

Further, the experimental data from different distances were statistically analyzed. The relationship between load resistance and transmission power under different transmission distances is obtained, as shown in FIG 12.

Analyzing FIG. 12, it shows that the transmission power increases firstly and then decreases with the increase of distance. For this experiment, when the transmission distance is $4.5 \mathrm{~cm}$, the transmission power is always maintained at the maximum value of different distances under the same resistance. The above figure shows the trend that the coupling coefficient decreases with the increase of transmission distance. At the same time, the output power increases firstly and then decreases with the increase of coupling coefficient. The experimental results are in agreement with the results of the previous theoretical analysis. 


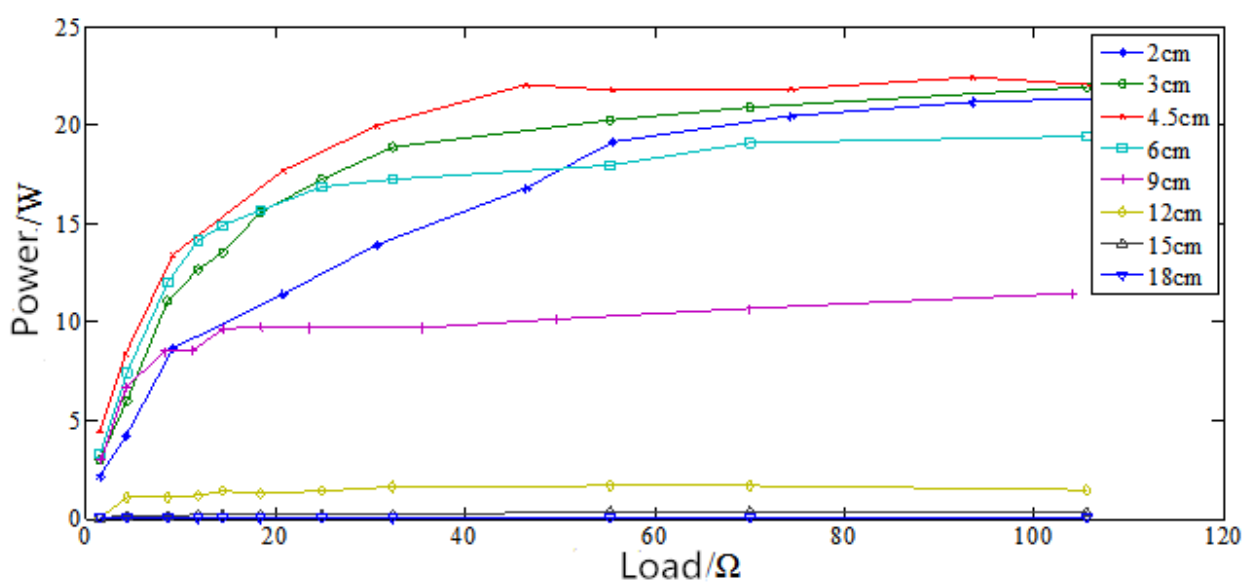

Fig. 12 Relationship between Load Resistance and Transmission Power under Different Transmission Distances

\section{Summary}

In this study, we studied the characteristics of RWPT in detail, and the relationship between the impedance, frequency and power of the system is analyzed with the help of mathCAD software, and the whole system is analyzed with the aid of PSpice software. Finally, the experimental system is built to investigate the transmission power of the system under the resonance frequency of $20 \mathrm{kHz}$, and the consistency between the theoretical analysis and the actual experiment is verified. At the same time, it lays the foundation for the next step of battery charging and energy feedback processing.

\section{Acknowledgements}

Scientific Innovation Practice Project of Postgraduates of Chang'an University(2018064).

\section{Reference}

[1]. Kurs A, Karalis A, Moffatt R, et al. Wireless power transfer via strongly coupled magnetic resonances [J]. Science, 2007, 317 (5834): 83-86.

[2]. Jianliang Li, Jing Kang, Chenglin Tian, et al. Study on Wireless Power Transfer Technology with Series-Series Type of Magnetic Coupling Resonance Model[A]. Proceedings of the 2nd International Conference on Computer Engineering, Information Science and Internet Technology (CII 2017) [C].Wuhan, China, 2017 . 225-232.

[3]. Li Jianliang, Cheng Bo. Study on a Wireless Power Transfer System with the Series-Series Type of Magnetic Coupling Resonance [J]. Journal of Shaoyang University (Natural Science Edition), 2018, 15 (02):46-54(in Chinese).

[4]. 4. Wenxian Chen, Qianhong Chen, Juntao Huang, et al. Analysis and research of distance transmission characteristics of magnetic resonance WPT system [A] . 2014 IEEE VTC [C]. Seoul, Korea, 2014. 1-5.

[5]. V. B. Gore and D. H. Gawali, "Wireless power transfer technology for medical applications," 2016 Conference on Advances in Signal Processing (CASP), Pune, India, 2016, pp. 455-460. 